\title{
Vertical Flow in the Thermoelectric Liquid Metal Plasma Facing Structures (TELS) facility at Illinois
}

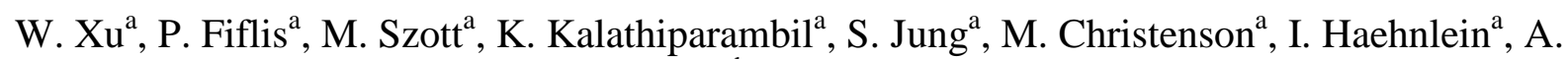 \\ Kapat $^{\mathrm{a}}$, D. Andruczyk ${ }^{\mathrm{a}, \mathrm{b}}$, D. Curreli ${ }^{\mathrm{a}}$ and D.N. Ruzic ${ }^{\mathrm{a}}$ \\ ${ }^{a}$ Center for Plasma-Material Interaction, Dept. Nuclear, Plasma, and Radiological Engineering, \\ University of Illinois at Urbana-Champaign, Urbana, USA \\ ${ }^{b}$ Research Engineer Stationed at PPPL
}

\begin{abstract}
Flowing liquid metal PFCs may offer a solution to the issues faced by solid divertor materials in tokamak plasmas. The Liquid-Metal Infused Trenches (LiMIT) concept of Illinois [1] is a liquid metal plasma facing structure which employs thermoelectric magnetohydrodynamic (TEMHD) effects to self-propel lithium through a series of trenches. The combination of an incident heat flux and a magnetic field provide the driving mechanism. Tests have yielded experimental lithium velocities under different magnetic fields, which agree well with theoretical predictions [2]. The thermoelectric force is expected to overcome gravity and be able to drive lithium flow along an arbitrary direction and the strong surface tension of liquid lithium is believed to maintain the surface when Li flows in open trenches. This paper discusses the behavior of the LiMIT structure when inclined to an arbitrary angle with respect to the horizontal.
\end{abstract}

PACS: 52.40.Hf, 52.55.Rk

PSI 20 Keywords: Divertor material, Lithium, Liquid metal

*Corresponding author address: 201 S. Goodwin, Rm. 105, Urbana, IL, USA

*Corresponding author email: fiflis1@illinois.edu

Presenting author: Kishor Kalathiparmabil 


\section{Introduction}

As the development of the tokamak progresses, enhancement of plasma and heat fluxes in the divertor region has placed increasing strain on existing plasma facing components. Solid divertor materials have begun to show deficiencies such as erosion [3], melting [4], and nanostructure formation [5]. Liquid materials have been proposed as a potential solution to this problem, in particular, liquid lithium [6]. Liquid lithium suffers from none of these deficiencies, and as a result may provide better power handling than tungsten. Lithium PFCs also offer the ability to reduce edge recycling $[7,8]$, resulting in a hotter edge region, increasing the volume of the fusion device at fusion temperatures [9]. Lithium has been shown to aid in suppressing ELMs [10], and to enhance plasma performance $[11,12,13]$. However, the higher vapor pressure would require maintaining lithium plasma facing components at a lower temperature, and while inclusion of lithium has been shown to reduce $\mathrm{Z}_{\mathrm{eff}}[14]$, events leading to significant evaporation of lithium would in turn beget core dilution.

A lithium PFC concept, LiMIT [1], has been designed and tested at Illinois. It is a self-driven liquid metal divertor alternative driven by thermoelectric magnetohydrodynamics [15, 16]. Composed of a series of lithium filled trenches, LiMIT employs thermoelectric currents flowing within the lithium, interacting with the main toroidal field of a fusion device to drive flow. Plasma incident on the top surface of the trenches with cooling of the trenches from below establishes a temperature gradient. Due to the Seebeck effect, thermoelectric currents arise from the temperature gradient along the walls of the trenches. The interaction of these currents with the toroidal magnetic field generates a $\mathrm{JxB}$ force driving lithium flow (See Figure 1). While the concept has been tested both at Illinois [2] and in HT-7 [17], further testing of this concept has been twofold. Addition of heaters and coolant channels now allows for the active generation of a 
thermal gradient with lithium channels, creating lithium flow independently of heat from plasma. When the temperature gradient, and therefore the thermoelectric current, is high enough, the thermoelectric force is expected to overcome gravity, and, aided by the high surface tension of the liquid lithium film over the trenches, is able to drive lithium flow along an arbitrary direction which is absolutely necessary if the system is to be used as a PFC in a working tokamak. A design of the LiMIT concept with narrow trenches has been manufactured and tested. This paper discusses the latest study of the TEMHD driven flow at arbitrary inclination of the LiMIT structure performed at Illinois.

\section{Li Flow along Arbitrary Angle}

The orientation of the lithium trenches during the thermoelectric driven liquid lithium flow tests at Illinois and in HT-7 was horizontal. The influence of the gravity when the flow direction is not horizontal is not yet well characterized. However, when considering a LiMIT type PFC for a limiter, divertor target plate, or even the inner wall, it is necessary to investigate flow along an arbitrary angle. The operative question in this case is if the thermoelectric driven force can overcome forces retarding the motion of lithium such as flow impedance of the trenches, MHD damping, and the force of gravity in the rear channels. Since the viscosity of lithium is very low, and the driving force is provided from an MHD effect, these effects can be shown to have negligible impact [2]. To consider the effect of gravity, the following force balance is considered. If the trench is placed vertically and the TEMHD pumping is at standstill, the rising pressure gradient $\nabla p$ is given as [15]

$$
\nabla p=\frac{\sigma B P}{(1+C)} \nabla T
$$


If the thermoelectric force is to overcome gravity to drive the lithium, the rising pressure gradient due to TEMHD must be larger than the body force $\rho g$ by gravity; a relation that simplifies to:

$$
\nabla T>\frac{\rho g(1+C)}{\sigma B P}
$$

Here $\nabla T$ is the temperature gradient across the lithium channel. $\rho=508 \mathrm{~kg} / \mathrm{m}^{3}$ and $\sigma$ are, respectively, the density and electrical conductivity of lithium. $B$ is the transverse magnetic field and $g$ is the gravity. $P$ is the thermoelectric power between the pair of materials and $C=$ $(a \sigma) /\left(t \sigma_{W}\right)$, describes the wall impedance in comparison with that of the lithium. $a$ is the thickness of liquid channel and $t$ is the thickness of the wall. $\sigma_{W}$ is the electrical conductivity of the wall.

When the thickness of the liquid channel is the same as the wall thickness, the required temperature gradient as a function of magnetic field is plotted in Figure 2 for different material pairs. The thermoelectric power values are taken from $[18,19]$. For the experiments at Illinois with $0.111 \mathrm{~T}$ transverse magnetic field the necessary temperature gradient is around $1.4 \times 10^{3}$ $\mathrm{K} / \mathrm{m}$, which is achieved via a temperature difference of a few tens of Kelvin over several millimeters, while for experiments in real fusion devices with much stronger magnetic fields, such as EAST, a temperature gradient of only about $45 \mathrm{~K} / \mathrm{m}$ should be enough to provide flow. Physical properties of liquid lithium are taken from Hanford report [20] assuming T=523 K. In the experiments described here, direct measurement of the thermal gradient in the liquid channels is difficult, and is therefore calculated from a 3D, time dependent model implemented in the finite element software, COMSOL, balancing heat in from the heater with heat lost to the coolant. Temperature values are averaged across the top surface and bottom surface of the lithium channels to determine the average temperature gradient in the channels. Both average temperature values, and the temperature gradient are plotted as the function of time in Figure 3. 
From this figure we can see that after about 10 seconds the temperature gradient changes little. An averaged value $2665 \pm 500 \mathrm{~K} / \mathrm{m}$ is chosen for further calculation.

When the trench is tilted to angle $\theta$ with respect to the horizontal direction, the mean velocity of the front side trenches is:

$$
u_{m_{-} \text {front }}=\frac{2}{3} \frac{H a-\tanh (H a)}{H a+C \tanh (H a)} \frac{P}{B} \frac{d T}{d y}
$$

which is equal to $0.114 \pm .021 \mathrm{~m} / \mathrm{s}$ for this case. Here the Hartmann number is $\mathrm{Ha}=\mathrm{B} \mathrm{a}$ / $2 \sqrt{ }(\sigma / \mu)$ and $\mu$ is the dynamic viscosity of liquid lithium. This result implies that the flow velocity should be independent of the inclination angle.

\section{Experimental Design and Setup}

To investigate the flow within the LiMIT structure at arbitrary inclination angle, a vacuum chamber was constructed on a pivot whose axis was the center of the LiMIT structure. The entire chamber was rotatable about this central pivot. The base pressure is kept below $1 \mathrm{mBar}$ with the help of a dry pump. A magnetic field of about $0.111 \mathrm{~T}$ at the LiMIT structure position is generated by powering four magnet coils with car batteries. The LiMIT channels in this case were $0.5 \mathrm{~mm}$ wide by $1 \mathrm{~mm}$ deep on front side and $0.5 \mathrm{~mm}$ wide by $2 \mathrm{~mm}$ deep on back side. A small tray is attached to one end to catch residual lithium. The whole structure is cooled by compressed air flowing through five $1 / 4$ " diameter holes at center and heated on the back side with a Watlow strip heater $(20 \mathrm{~cm}$ by $4 \mathrm{~cm}$ by $0.3 \mathrm{~cm}$ ) with a maximum power of $500 \mathrm{~W}$ at $120 \mathrm{~V}$. Two thermocouples are attached to the front side, close to the edge of the front trenches while one thermocouple is attached to the heater surface. The heater is attached to the backside of LiMIT by four stainless steel clamps with approximately $0.05 \mathrm{~mm}$ thick UHV high temperature carbon paste at the interface. 
Filling of these channels was accomplished with a lithium injector similar to that used in [21] with the addition of a $1 \mathrm{~mm}$ inner diameter needle type nozzle and two linear shifts. A schematic is shown in Figure [1]. The nozzle was lowered via the linear shift into a space on the side of the trenches and lithium was injected filling the trenches from the bottom up. Once the trenches were filled the nozzle was lifted above the lithium surface. Although lithium is observed to start to wet untreated stainless steel surface at around $330{ }^{\circ} \mathrm{C}$ [22] lithium was melted into these narrow channels by a temperature excursion of the structure to about $475^{\circ} \mathrm{C}$.

To investigate lithium flow rate at arbitrary inclination, a thermal gradient was established by maintaining the LiMIT structure at $400{ }^{\circ} \mathrm{C}$ steady state and the heater surface at $600{ }^{\circ} \mathrm{C}$, without flowing coolant. Compressed air was then flowed at $50 \mathrm{slm}$ at 2 bar, resulting in a reduction in temperature of the device, but producing a higher transient temperature gradient than what the heater and coolant can provide at steady state. The magnetic field was then toggled on and the chamber was inclined to the desired angle while recording with a high speed camera (Phantom v9.1, Vision Research). Velocity of the lithium was extracted from frame to frame displacement of lithium hydroxide and oxide impurities entrained in the lithium flow. Due to resolution limitations, tracking of these impurities in the raw images was difficult and a Java-based image processing program, ImageJ, was employed to increase the contrast of each image. Impurity scale is formed primarily from the gettering of residual water vapor in the chamber over the course of several minutes. Impurity formation in a fusion device would occur over a much longer time scale, as the base pressure (1e- 8 mbar) is much lower than the base pressure of the chamber used for these experiments (1e-3 mbar).

In the experiments detailed herein it was observed that trenches are completely filled and capillary force was strong enough to maintain the surface from horizontal to vertical orientation. 
Even when the trenches were overfilled the residual lithium would just fall into the tray at one end and the trenches still remained filled. This is shown in Figure 4.

\section{Results and Discussion}

Flow velocity measurements with LiMIT aligned horizontally were taken in the manner described above. A series of frames processed using ImageJ are shown in Figure 5. In total, 12 clips of moving impurity scale are analyzed giving an average velocity of $0.095+/-0.041 \mathrm{~m} / \mathrm{s}$. The lithium trenches were subsequently inclined to a series of angles between 0 and 90 degrees from the horizontal. The velocities of the lithium were similarly extracted and the results can be seen in Figure 6. Also plotted within Figure 6 are the theoretical prediction for the velocity, as well as error bars resulting from the uncertainty in the determination of the thermal gradient in the rear channels. Good agreement can be seen between the theoretical prediction and the experimental velocity at higher inclination angles. Poorer agreement is seen at lower angles, even though in most cases there is overlap of the error bars. The general trend however, appears to be increasing from low inclination to high inclination, even though the gravitational force is predicted to be small compared to gravity. This is attributed to an overfill layer seen in LiMIT structure. At low inclination angles, the overfill is spread over the entire LiMIT tray, whereas at large inclination angles, the overfill was collected at the bottom of the channels. Since velocity measurements were taken near the center of the structure, the overfill would lead to an increased cross sectional area, and due to conservation of flow rate, a lower velocity. A ratio of velocities implies an overfill of approximately $1 \mathrm{~mm}$ in the cases of low inclination angle. Evidence of such an overfill can be seen in Figure 4.

\section{Conclusion}


The concept of TEMHD driven liquid lithium flow along an arbitrary direction has been implemented and tested at Illinois. Several predictions were validated. First, surface tension is able to maintain the fluid surface even against the hydrostatic pressure of aligning a LiMIT device vertically. Also, TEMHD lithium flow is able to overcome gravity to drive flow vertically, and theoretical predictions of the independence of velocity on inclination angle showed decent agreement with experimental results. These experiments have demonstrated the feasibility of this type of flow. The ability to utilize the LiMIT concept for all surfaces in a future fusion reactor is an important step toward realizing a flowing metal wall scenario.

\section{Acknowldegements}

The authors would like to recognize the US DOE which funds this work under project DE-FOA0000603.

\section{References}

[1] D.N. Ruzic, W. Xu, D. Andruczyk, M.A. Jaworski. Lithium-metal infused trenches (LiMIT) for heat removal in fusion devices. Nucl. Fusion 51 (2011) 10200

[2] W. Xu, D, Curreli, D. Andruczyk, T. Mui, R. Switts and D.N. Ruzic, Heat transfer of TEMHD driven lithium flow in stainless steel trenches. J. Nuclear Material, 438 (2013) S422

[3] J.N. Brooks, J.P. Allain, R.P. Doerner, A. Hassanein, R. Nygren, T.D. Rognlien, and D.G. Whyte. Plasma-surface interaction issues of an all-metal ITER. Nucl. Fusion 49 (2009) 035007

[4] J.W. Coenen, G. Arnoux, B. Bazylev, G.F. Matthews, S. Jachmich, I. Balboa, M. Clever, R. Dejarnac, I. Coffey, Y. Corre, S. Devaux, L. Frassinetti, E. Gauthier, J. Horacek, M. Knaup, M. Komm, K. Krieger, S. Marsen, A. Meigs, Ph. Mertens, R.A. Pitts, et al. ELM induced tungsten melting and its impact on tokamak operation. J. Nuc. Mat. In Press.

[5] M.J. Baldwin and R.P. Doerner. Helium induced nanoscopic morphology on tungsten under fusion relevant plasma conditions. Nucl. Fusion 48 (2008) 035001 
[6] M.A. Abdou and et al, On the exploration of innovative concepts for fusion chamber technology, Fusion Engineering and Design 54 (2001) 181

[7] J. A. Snipes et. al. Wall conditioning with impurity pellet injection on TFTR J. Nuclear Material, 196 - 198 (1992) 686

[8] R. A. Pitts, et. al., ELM transport in the JET scrape-off layer, Nuclear Fusion, 47 (2007) 1437

[9] D. N. Ruzic, M. C. Allain, R. V. Bundy, The effect of lithium wall conditioning in TFTR on plasma-surface interactions, J. Nuclear Material, 266 - 269 (1999) 1303

[10] J. Kallman, M. A. Jaworski, R. Kaita, H. Kugel, T. K. Gray, High density Langmuir probe array for NSTX scrape-off layer measurements under lithiated diverter conditions, Rev. Sci. Instrum. 81 (2010) 10E117

[11] S. V. Mirnov, Plasma wall interactions and pasma behavior in fusion devices with liquid lithium plasma facing components, J. Nuclear Material, 390 - 391 (2009) 876

[12] A. Vertkov et. al., Technological aspects of liquid lithium limiter experiment on FTU tokamak, Fusion Engineering and Design, 82 (2007) 1627

[13] R. Majeski et. al. Performance projections for the lithium tokamak experiment, Nuclear Fusion, 49 (2009) 055014

[14] G. Mazzitelli, M.L. Apicella, V. Pericoli Ridolfini, G. Apruzzese, R. De Angelis, D. Frigione, E. Giovannozzi, L. Gabellieri, G. Granucci, C. Mazzotta, M. Marinucci, A. Romano, O. Tudisco, A. Alekseyev, I. Ljublinski, A. Vertkov, ECRH Team. Review of FTU results with the liquid lithium limiter. Fusion Engineering and Deign, 85 (2010) 896.

[15] J. A. Shercliff, Thermoelectric magnetohydrodynamics. Journal of Fluid Mechanics, 91:231-251, 1979

[16] M. A. Jaworski, T. K. Gray, M. Antonelli, J. J. Kim, C.Y. Lau, M. B. Lee, M. J. Neumann, W. Xu, and D. N. Ruzic, Thermoelectric Magnetohydrodynamic Stirring of Liquid Metals, PRL 104, 094503 (2010)

[17] J. Ren and et al, First results of flowing liquid lithium limiter in HT-7, Phys. Scr. T159 (2014) 014033 (5pp)

[18] V. Surla, M. Tung, W. Xu, D. Andruczyk, M. Neumann, D. N. Ruzic and D. Mansfield, "Seebeck Coefficient Measurements of Lithium Isotopes“, J. Nuc. Matter 415 (2011) 18-22.

[19] P. Fiflis, L. Kirsch, D. Andruczyk, D. Curreli, D.N. Ruzic , "Seebeck coefficient measurements on Li, Sn, Ta, Mo, and W', Journal of Nuclear Materials 438 (2013) 224-227 
[20] D.W. Jeppson, J. L Ballif, W.W. Yuan, and B. E. Chou, Lithium literature review: Lithium's properties and interactions. Technical Report HEDLTME 78-15, Hanford Engineering Development Laboratory, 1978

[21] P. Fiflis, D. Andrucyzk, A. L. Roquemore, M. McGuire, D. Curreli, and D. N. Ruzic, "Lithium pellet produciton (LiPP): A device for the production of small spheres of lithium", Review of Scientific Instruments, Vol. 84, No. 6, 2013.

[22] P. Fiflis, A. Press, W. Xu, D. Andruczyk, D. Curreli, D.N. Ruzic. Wetting Properties of Liquid Lithium on Select Fusion Relevant Surfaces. Fus. Eng. Des. In Press (2014)

\section{Figure captions}

Figure 1: LiMIT structure cross section and schematic of filling procedure for arbitrary inclination of TEMHD driven flow test illustrating the lithium channels, driving JxB forces, and filling mechanism.

Figure 2: Required temperature gradient to overcome gravity vs magnetic field. Points added illustrating location in parameter space of recent Illinois tests and prediction of location of EAST module under design.

Figure 3: 3D time dependent heat transfer model result illustrating calculated average temperatures and temperature gradient across the back side channels as a function of time. After 10 s, gradient is approximately constant at $\sim 2650 \mathrm{~K} / \mathrm{m}$.

Figure 4: Top view LiMIT module used in arbitrary inclination tests showing empty lithium trenches (above) and filled lithium trenches (below). Capillary force is able to maintain the surface no matter how the trenches are tilted.

Figure 5: Series of frames from fast frame camera illustrating the moving impurity scales. Frame to frame impurity displacement is used to determine the velocity of the lithium. Images shown have been processed with ImageJ to increase contrast. 
Figure 6: Lithium velocity as a function of inclination angle, higher inclination angles show better agreement with theoretical prediction. Lower inclination angles have a lower measured velocity than theoretically predicted, which is hypothesized to be due to overfill.

\section{Figures}

- Allows for lithium filling from the bottom up

- Bellows give sufficient range of motion to allow the dropper to be moved away from the system during testing

- Small tip mitigates wetting problem during injection
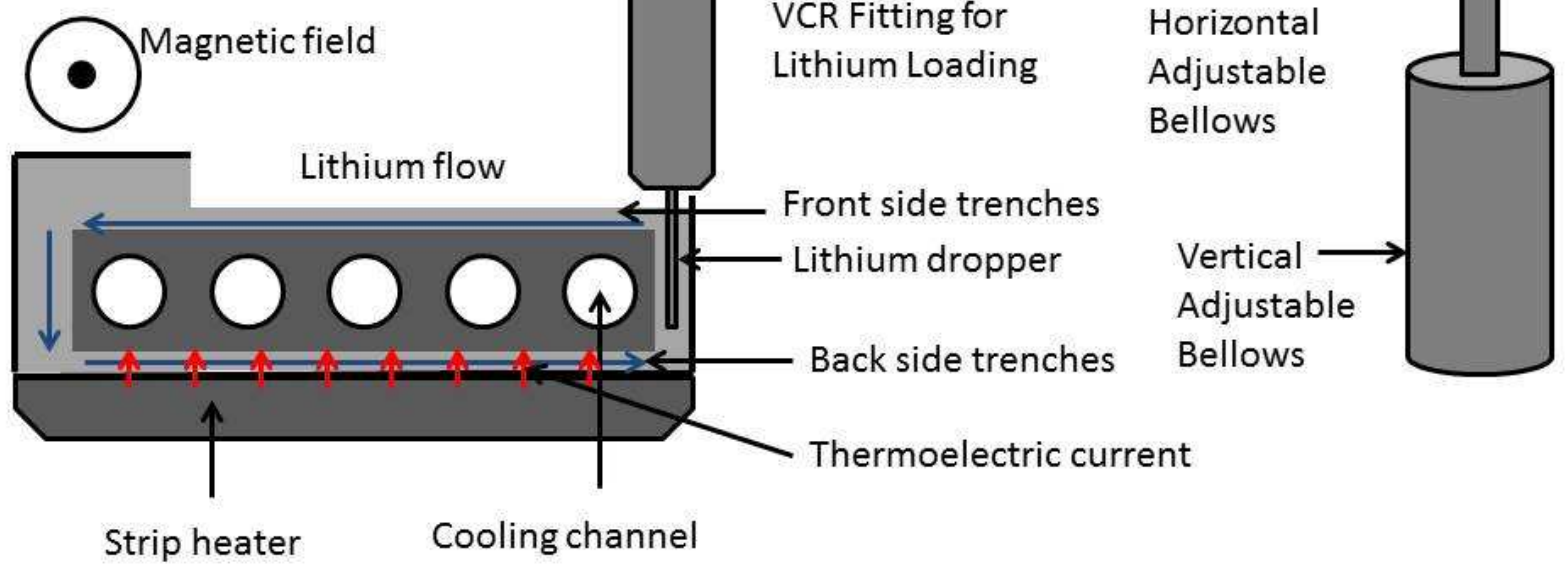

Figure 1 


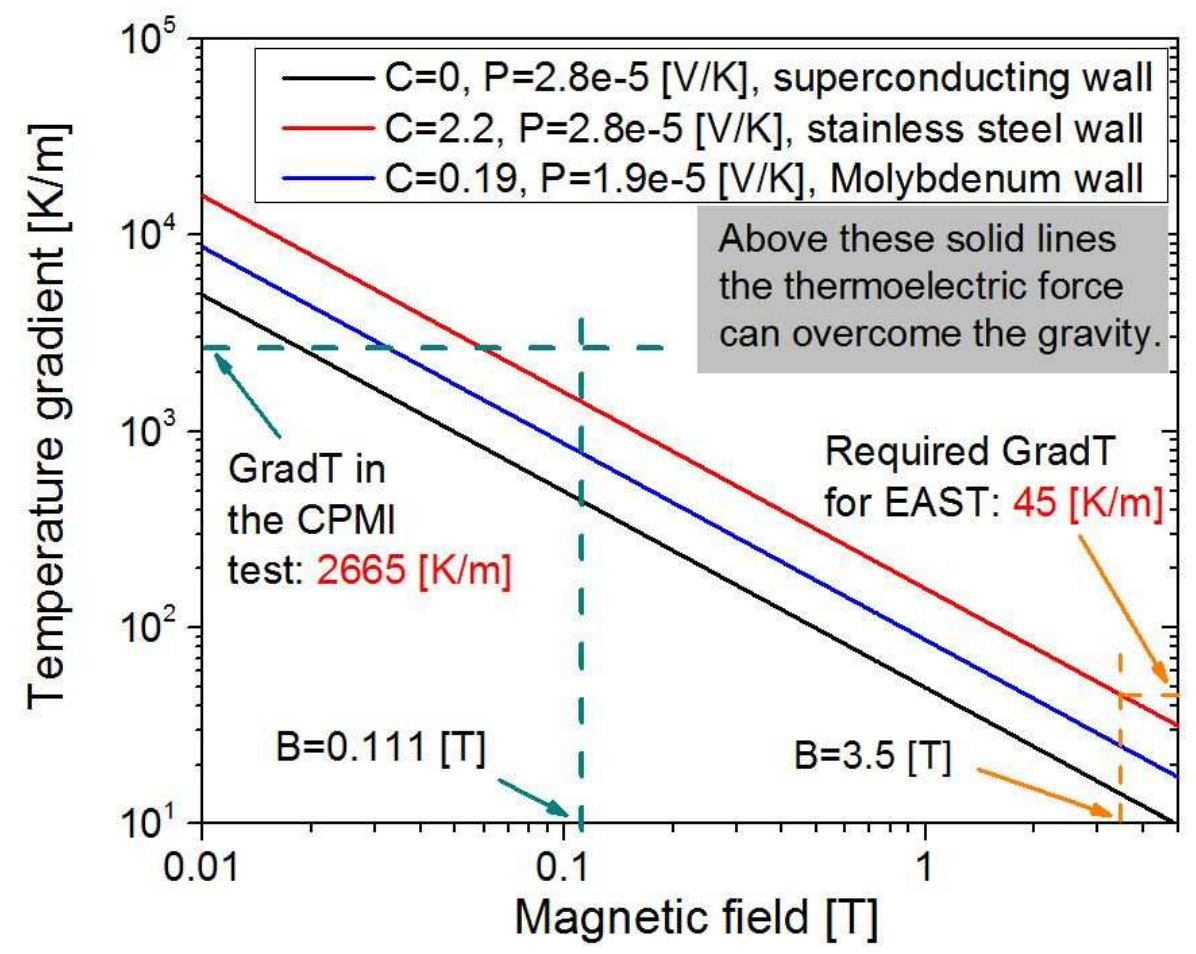

Figure 2 


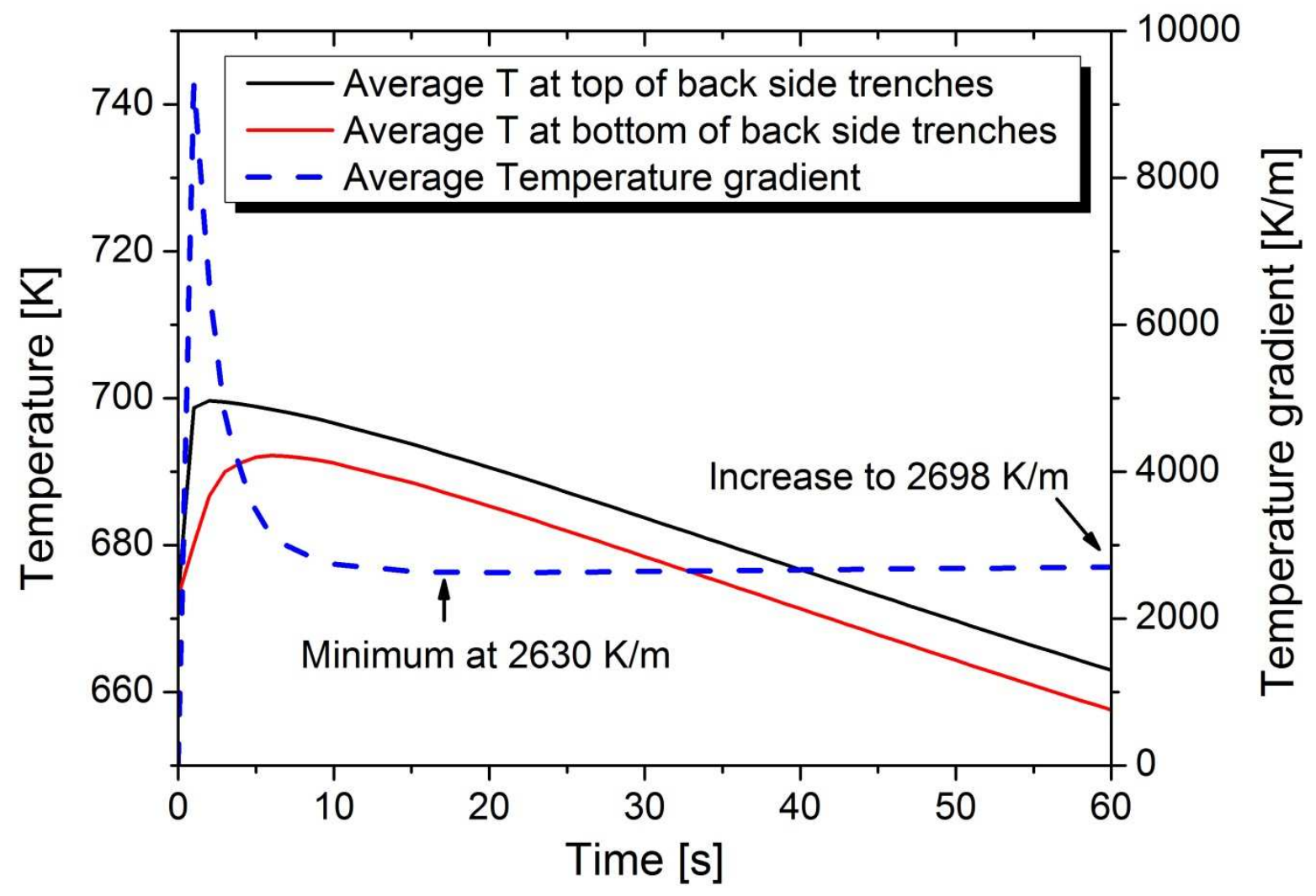

Figure 3 


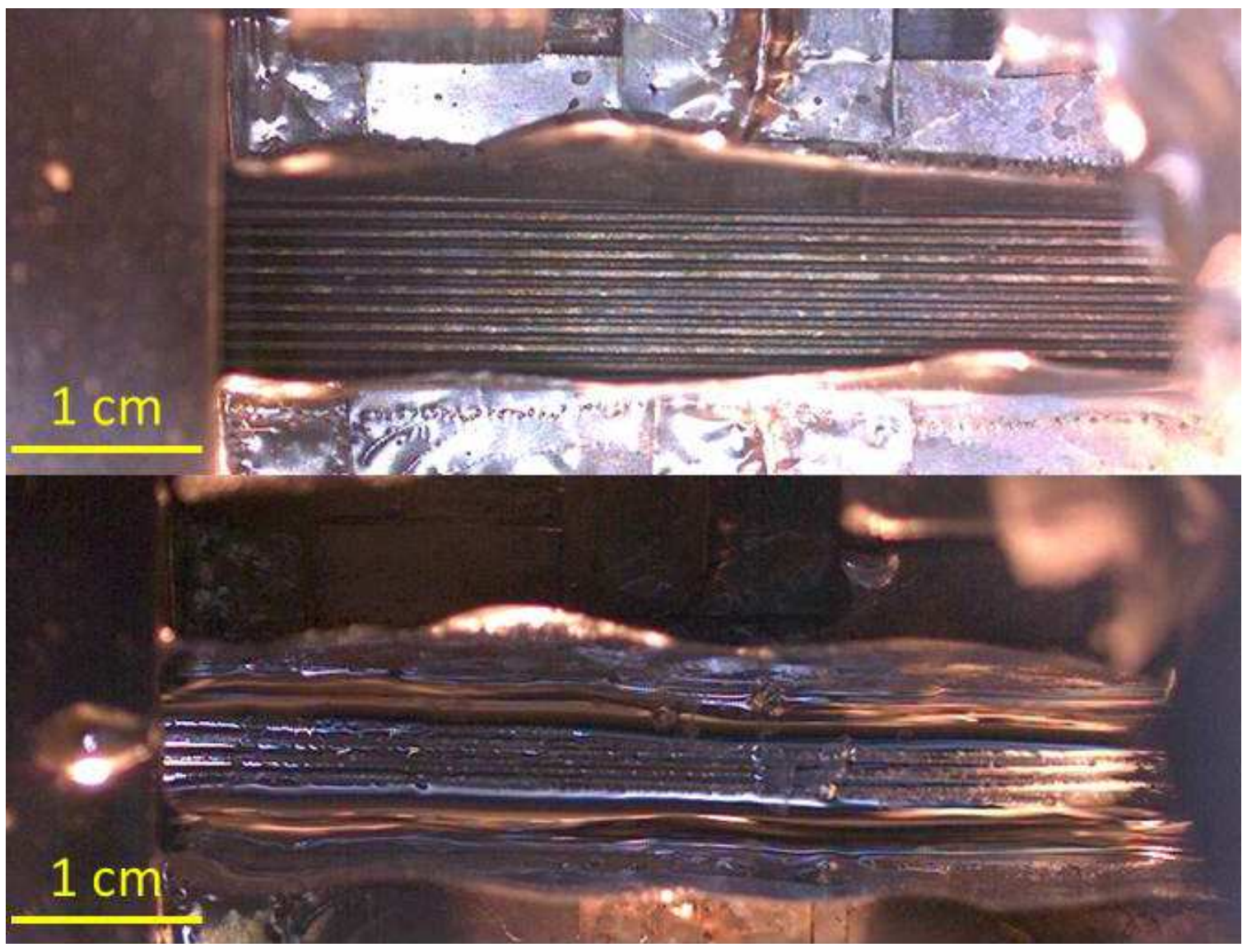

Figure 4 


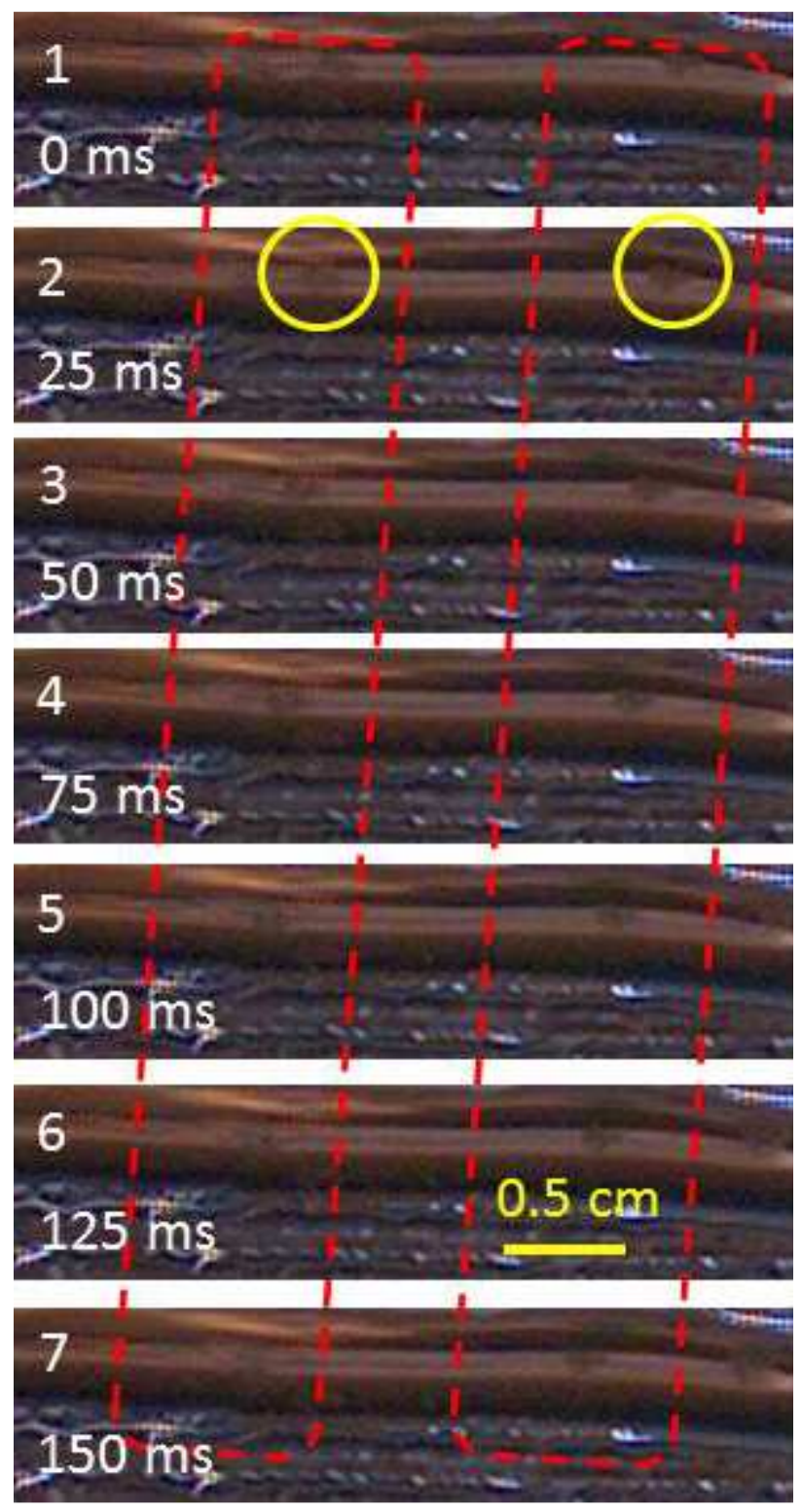

Figure 5 


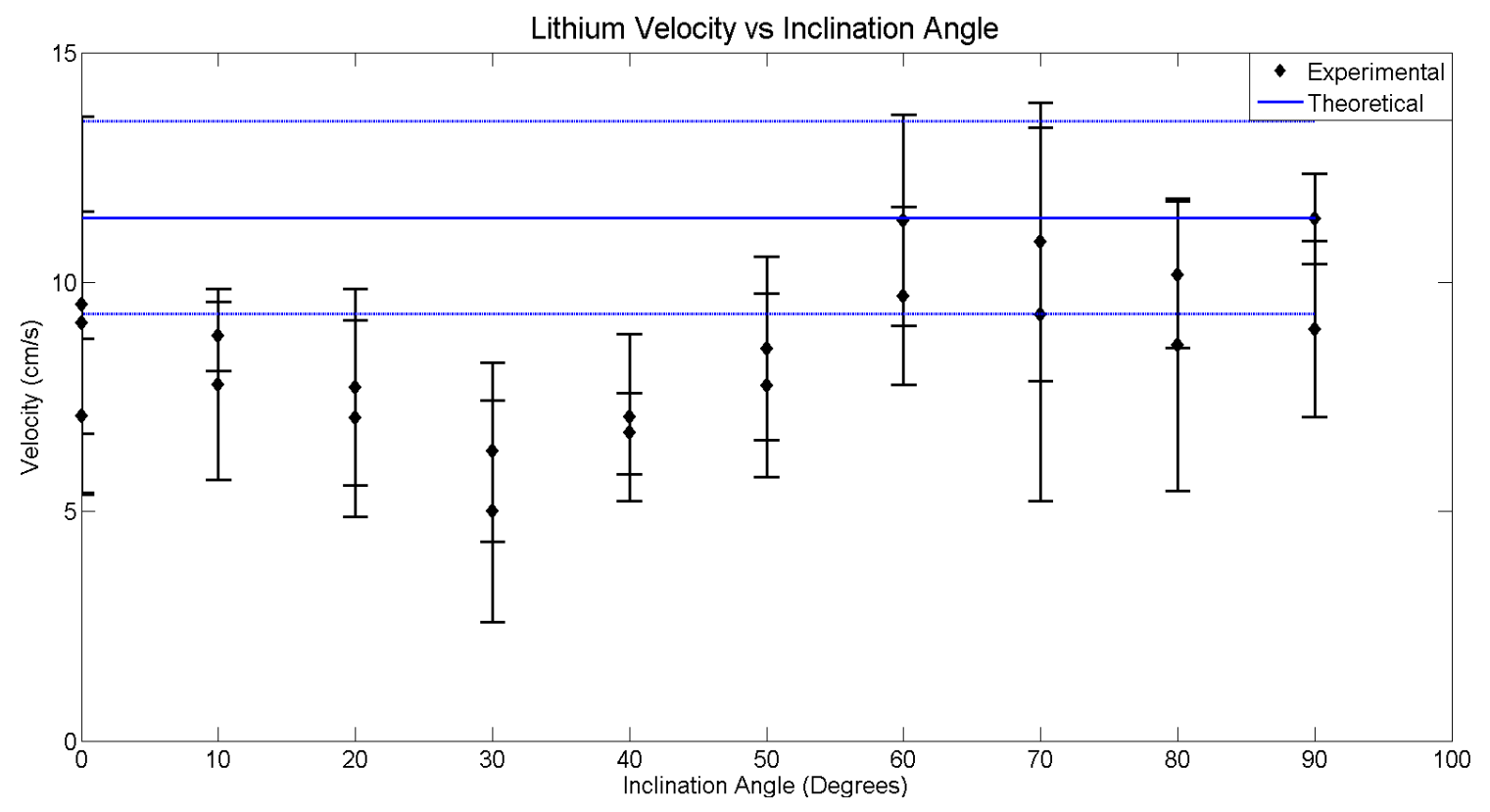

Figure 6 